\title{
ORIGINAL ARTICLE \\ Physical impairment and walking function required for community ambulation in patients with cervical incomplete spinal cord injury
}

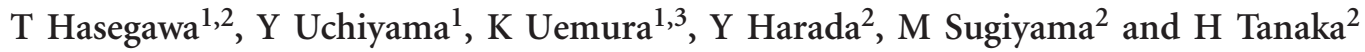

Study design: Cross-sectional study.

Objective: To identify the physical impairments and walking function required for community ambulation in patients with cervical incomplete spinal cord injury (ISCI).

Setting: Chubu Rosai Hospital, Nagoya, Japan.

Methods: Forty patients with cervical ISCI (mean age: 49.9 years, American Spinal Injury Association Impairment Scale D) were included. The primary outcome measure was community ambulation based on Spinal Cord Independence Measure outdoor scores for a distance of $>480 \mathrm{~m}$. We measured the upper- and lower-extremity motor scores (UEMS and LEMS), sensory and spasticity. The walking tests included $10 \mathrm{~m}$ of walking at a comfortable- and maximum-walking speed (CWS and MWS; $\mathrm{ms}^{-1}$ ), 6 min walking test (6MWT; $\mathrm{m}$ ) and the walking index for spinal cord injury II (WISCI II). Multivariate logistic regression models were used to assess the physical impairments associated with community ambulation. Receiver operating characteristic curves were analyzed to determine the cutoff points for physical impairment and walking function.

Result: The LEMS (beta coefficient $(\beta)=0.71$ ) and UEMS ( $\beta=0.41$ ) were independently associated with community ambulation in patients with cervical ISCI. The cutoff points of the LEMS, UEMS, CWS, MWS, 6MWT and WISCI II were 41.5, 36.5, $1.00 \mathrm{~m} \mathrm{~s}^{-1}$, $1.32 \mathrm{~m} \mathrm{~s}^{-1}, 472.5 \mathrm{~m}$ and 17.5 , respectively, which suggests moderate to high accuracy.

Conclusion: The LEMS and UEMS were the most important factors affecting community ambulation in patients with cervical ISCI. The cutoff points of the walking function tests were highly accurate; therefore, these points can serve as targets for walking training in the future.

Spinal Cord (2014) 52, 396-399; doi:10.1038/sc.2014.18; published online 11 March 2014

Keywords: WISCI; walking speed; 6MWT; strength; SCIM

\section{INTRODUCTION}

Recent statistics indicate that the proportion of cervical and neurologically incomplete injuries is increasing in patients with spinal cord injury. ${ }^{1}$ The recovery rates of American Spinal Injury Association motor scores for incomplete tetraplegia are greater than those for incomplete paraplegia; however, patients with incomplete tetraplegia are less likely to walk than those with incomplete paraplegia. ${ }^{2}$ Therefore, recovering the ability to walk is an important goal for patients with cervical incomplete spinal cord injury (ISCI) and has become a target of several rehabilitative approaches. Moreover, precisely evaluating walking in these patients has become crucial. ${ }^{3}$ In recent years, this emphasis has been extended to include the attainment of community ambulation as an important mobility and social outcome. ${ }^{4}$

Community ambulation has been defined as the ability 'to ambulate and to achieve independence within the community, which includes skills such as crossing streets with traffic lights, shopping, walking at specified velocities and walking long distances. ${ }^{5}$ The American Spinal Injury Association impairment scale (AIS) D patients have a good prognosis for walking, but not all AIS D patients can achieve community ambulation. ${ }^{4}$
Attempts to evaluate community ambulation are currently underway. Physical impairment is an important determinant of community ambulation. Previous studies have shown a correlation between walking function and age, muscle strength, spasticity, sensory and balance in patients with ISCI. ${ }^{6,7}$ However, few comprehensive studies have shown the relationship between physical impairment and community ambulation in patients with cervical ISCI. Identifying the most relevant physical impairments associated with community ambulation, and determining the cutoff point of physical impairment, may help establish effective treatments for such patients.

Previous studies have already shown that walking speed and endurance are good indicators of community ambulation. ${ }^{8}$ Walking function in patients with ISCI is characterized by a slower walking speed and poor efficiency. ${ }^{4}$ van Hedel et al. ${ }^{9}$ suggested that a minimum walking speed of $0.44 \mathrm{~ms}^{-1}$ using a walking device resulted in independent community ambulation in patients with ISCI. Brotherton et al. ${ }^{10}$ reported that the use of a single cane or crutch allowed patients with ISCI to have independent community ambulation. However, few studies have comprehensively examined the cutoff point of walking function required for community

${ }^{1}$ Department of Physical Therapy, Graduate School of Medicine, Nagoya University, Nagoya, Japan; ${ }^{2}$ Department of Rehabilitation Medicine, Chubu Rosai Hospital, Nagoya, Japan and ${ }^{3}$ Research Fellowship division, Japan Society for the Promotion of Science, Tokyo, Japan

Correspondence: Professor Y Uchiyama, Department of Physical Therapy, Graduate School of Medicine, Nagoya University, 1-1-20 Daikouminami, Higashi-ku, Nagoya 461-8673, Japan. E-mail: uchiyama@met.nagoya-u.ac.jp

Received 2 March 2013; revised 28 January 2014; accepted 3 February 2014; published online 11 March 2014 
ambulation, including walking endurance and the use of walking devices or braces, in patients with cervical ISCI. Determining the walking function cutoff point required for community ambulation may help set clear clinical goals for these patients.

Therefore, the objectives of this study were as follows: (1) to identify the physical impairments associated with community ambulation, and (2) to determine the cutoff points of physical impairments and walking function for community ambulation in patients with cervical ISCI.

\section{MATERIALS AND METHODS \\ Patients}

In patients with cervical ISCI who were graded according to the AIS D and were able to walk independently (with or without assistive devices) over a 10-m distance on a level surface were included in the study. We also included patients within the age range of $16 \times 70$ years. Patients with severe cognitive impairment, brain injury and orthopedic or neurological conditions in addition to ISCI were excluded. Patients were recruited from April 2010 to December 2012. Forty patients with cervical ISCI were recruited (three women; mean age, 49.9 years). The median time since injury was 142 days (range, 7-277 days). All patients had injury levels ranging from $\mathrm{C} 4$ to $\mathrm{C} 8$. In accordance with the Declaration of Helsinki, the patients were informed of the experimental procedure, and each patient provided written-informed consent before participating in the study. The experimental procedure was approved by the local ethics committee (Graduate School of Medicine, Nagoya University, approval no. 10-513).

\section{Community ambulation}

The ability to ambulate within the community was the primary functional outcome. The Spinal Cord Independence Measure (SCIM) $)^{11}$ is the only comprehensive ability rating scale designed specifically for patients with SCI. We applied a cutoff, the Spinal Cord Independence Measure mobility score to distinguish between individuals who could walk independently and those who could not, as previously reported. ${ }^{12}$ Patients with scores of $0-3$ were grouped and defined as individuals unable to walk or who required assistance, whereas the patients with scores of 4-8 were grouped and defined as individuals able to walk independently. van Hedel et al. ${ }^{9}$ used the Spinal Cord Independence Measure outdoor mobility scores to examine community ambulation. This score assesses the ability to walk $>100 \mathrm{~m}$, although Robinett and Vondran ${ }^{5}$ found that the community ambulation distances are often greater than the distance benchmarks used in clinical measurements. These authors suggested that individuals may have to ambulate as far as $480 \mathrm{~m}$ when visiting supermarkets. ${ }^{5}$ Therefore, our study defined community ambulation based on the Spinal Cord Independence Measure outdoor scores of 4-8, which indicate an ability to walk independently for a distance $>480 \mathrm{~m}$. As there was a supermarket $500 \mathrm{~m}$ away from our hospital, we assessed whether it was possible for patients to visit the supermarket independently using a route that included uneven terrain and curbs.

\section{Walking function}

Patients were subjected to a $10-\mathrm{m}$ walking test and a 6 -min walking test (6MWT; m). Patients wore sneakers that were individually fitted and used in their daily life, and utilized whatever assistive and/or orthotic devices they preferred. The $10-\mathrm{m}$ walking test was performed at both a comfortable- and maximum-walking speed. The test was performed with a dynamic start to allow a $2-\mathrm{m}$ acceleration, the timed $10-\mathrm{m}$ distance and a 2-m deceleration. Speed was only calculated for the $10-\mathrm{m}$ distance between the 'end zones'. The $6 \mathrm{MWT}$ was a measure of distance and represented the maximum distance walked in $6 \mathrm{~min}$. During the test, patients walked up and down a $46-\mathrm{m}$ rectangular walkway. A licensed and well-trained physical therapist assessed all of the physical performance tests. Walking level was assessed using the walking index for spinal cord injury II (WISCI II). ${ }^{13}$

\section{Physical impairment}

Neurological examinations were performed according to the international standards for the classification of SCI. ${ }^{14}$ A trained examiner assessed upperand lower-extremity motor scores (UEMS and LEMS), light touch scores (LTS) and pin-prick scores (PPS). Lower-extremity spasticity was measured using the composite-modified Ashworth Scale (CMAS). ${ }^{15}$ As published previously, this scale uses a $0-4$ score range with a $1+$ grade, but for data analyses the scores were adjusted to give a $0-5$ score range ( $1+$ became 2,2 became 3 and so on). This scale was applied to the knee and ankle flexors. The scores of both sides were added, resulting in a total CMAS score range of 0-20.

\section{Statistical analysis}

Descriptive analyses were used to calculate the percentage, mean and median values for several demographic variables including age, sex and time since injury. The normality of distribution was tested with the Shapiro-Wilk test. The differences between the independent and dependent community ambulation groups were analyzed using Student's $t$-tests (for the normally-distributed data) and Mann-Whitney $U$-tests (for nonnormally-distributed data). The relationship between community ambulation and patient characteristics was examined using Spearman's correlation. A linear regression analysis was performed to describe the relationship between LEMS, walking speed and 6MWT. The multivariate logistic regression model using a forward stepwise selection method was used to select the final set of independent factors for community ambulation. Independent variables included age, time since injury, UEMS, LEMS, LTS, PPS and CMAS. A significance level of $P<0.05$ was used for all statistical analyses. Curves for the receiver operating characteristics were inspected to determine the cutoff points for the UEMS, LEMS, LTS, PPS, CMAS, 6MWT, WISCI II and comfortable- and maximum-walking speed that best discriminated between independence and dependence in community ambulation. Cutoff points for maximizing the sensitivity and specificity for each test were determined using the Youden index. ${ }^{16}$ Data were analyzed using the Statistical Package for the Social Sciences for Windows (SPSS, version 19.0, Chicago, IL, USA).

\section{RESULTS}

Out of 40 patients, 37 were men (92.5\%). Five patients walked with one ankle-foot orthosis (AFO); one walked with two AFOs; and 34 walked without lower-extremity devices. Thirty-seven of the 40 patients were able to stand unsupported, and three patients were able to stand by using a hand for support.

Table 1 shows the differences in demographic factors, physical impairments and walking function between the independent and dependent community ambulation groups. Twenty-two patients (55\%) were independent, and $18(45 \%)$ were dependent. Table 2 shows the differences in WISCI II between the independent and dependent community ambulation groups. In the independent group, patients used a single cane, crutch or no device.

Univariate analyses showed significant correlations between community ambulation and age $(r=-0.32, P=0.044)$, time since injury $(r=0.31, P=0.048)$, UEMS $(r=0.54, P<0.01)$ and LEMS $(r=0.68$, $P<0.01)$. There were no significant correlations between community ambulation and sex, LTS, PPS or CMAS. Significant correlations also existed between the LEMS, walking speed and 6MWT $\left(r^{2}=0.57\right.$ and 0.63 , respectively; $P<0.01$ ).

Logistic regression analyses revealed that the LEMS $(\beta=0.71$; $P=0.008)$ and UEMS $(\beta=0.41 ; P=0.015)$ were independently associated with community ambulation. There were no statistically significant relationships between age, time since injury, LTS, PPS or CMAS. The receiver operating characteristics curve analyses are included in Table 3. The areas under the curve (AUC) of the LEMS, comfortable- and maximum-walking speed, 6MWT and WISCI II ranged from 0.90 to 0.96 , suggested high accuracy. The AUC of the 
Table 1 Comparison of demographic factors, physical impairments and walking function between the independent and dependent community ambulation groups

\begin{tabular}{|c|c|c|c|}
\hline \multirow[t]{2}{*}{ Variables } & \multicolumn{2}{|c|}{ Group } & \multirow[t]{2}{*}{ P-value } \\
\hline & $\begin{array}{l}\text { Independent } \\
\quad(\mathrm{n}=22)\end{array}$ & $\begin{array}{l}\text { Dependent } \\
(\mathrm{n}=18)\end{array}$ & \\
\hline Age (years) ${ }^{\mathrm{a}}$ & $45.9(12.0)$ & $54.7(12.8)$ & $0.031^{\mathrm{b}}$ \\
\hline \multicolumn{4}{|l|}{ Sex } \\
\hline Men $(n)$ & 21 & 16 & \\
\hline Women $(n)$ & 1 & 2 & \\
\hline Time since injury (days) & $122.7(57.2)$ & $158.8(71.9)$ & $0.085^{b}$ \\
\hline Upper-extremity motor score (points) ${ }^{c}$ & $46.0(7.0)$ & $35.5(8.0)$ & $<0.001^{d}$ \\
\hline Lower-extremity motor score (points) ${ }^{c}$ & $47.0(4.0)$ & $39.5(5.0)$ & $<0.001^{d}$ \\
\hline $\begin{array}{l}\text { Composite-modified Ashworth } \\
\text { scale (points)c }\end{array}$ & $8.0(4.0)$ & $9.0(4.0)$ & $0.410^{d}$ \\
\hline Light touch score (points) ${ }^{c}$ & $81.0(23.0)$ & $77.5(22.0)$ & $0.670^{d}$ \\
\hline Pin-prick score (points) ${ }^{c}$ & $78.5(26.0)$ & $80.5(21.0)$ & $0.947^{d}$ \\
\hline Comfortable-walking speed $\left(\mathrm{m} \mathrm{s}^{-1}\right)^{\mathrm{a}}$ & $1.2(0.3)$ & $0.8(0.2)$ & $<0.001^{b}$ \\
\hline Maximum-walking speed $\left(\mathrm{ms}^{-1}\right)^{\mathrm{a}}$ & $1.7(0.4)$ & $1.1(0.3)$ & $<0.001^{b}$ \\
\hline 6-min walking test $(\mathrm{m})^{\mathrm{a}}$ & $529.8(102.8)$ & $332.4(79.8)$ & $<0.001^{b}$ \\
\hline Walking index for spinal cord injury $\|^{c}$ & $20.0(1.0)$ & $13.0(0.0)$ & $<0.001^{d}$ \\
\hline
\end{tabular}

Values are shown as the mean (s.d.) for normally-distributed data.

${ }^{\mathrm{b}} P$-value from Student's $t$-test.

${ }^{\mathrm{C} V a l u e s}$ are shown as the median (interquartile range) for nonnormally-distributed data.

d $P$-value from Mann-Whitney U-test.

Table 2 Comparison of WISCI II between the independent and dependent community ambulation groups

\begin{tabular}{lcc} 
WISCI II & \multicolumn{2}{c}{ Group } \\
\cline { 2 - 3 } & Independent $(\mathrm{n}=22)$ & 2 \\
& & 1 \\
9 & & 11 \\
12 & 2 & 2 \\
13 & & 2 \\
15 & 7 & \\
16 & 13 & \\
19 & & \\
20 & & \\
\hline
\end{tabular}

Abbreviation: WISCI II, Walking index for spinal cord injury II.

UEMS was 0.85 , which suggested a moderate accuracy, while the AUC of the CMAS, LTS and PPS suggested a low accuracy.

\section{DISCUSSION}

We comprehensively investigated the physical impairments associated with community ambulation and determined the cutoff points of physical impairments and walking function required for community ambulation in patients with cervical ISCI. The results of this study revealed that the LEMS and UEMS were the most important factors affecting community ambulation, and the cutoff points of walking function were highly accurate.

Lower-extremity muscle strength was the most important factor for community ambulation in patients with cervical ISCI. This is in line with studies showing a correlation between the LEMS and walking function in patients with ISCI. ${ }^{6,17}$ On the other hand, Buehner et al. ${ }^{18}$ suggested that changes in the LEMS do not capture the full extent of
Table 3 Cutoff points of physical impairments and walking function for community ambulation

\begin{tabular}{|c|c|c|c|c|}
\hline Variables & $\begin{array}{l}\text { Cutoff } \\
\text { point }\end{array}$ & Sensitivity & Specificity & $A \cup C$ \\
\hline Upper-extremity motor score (points) & 36.5 & 0.91 & 0.67 & 0.85 \\
\hline Lower-extremity motor score (points) & 41.5 & 0.91 & 0.89 & 0.92 \\
\hline Composite-modified Ashworth scale (points) & 10.5 & 0.05 & 0.94 & 0.39 \\
\hline Light touch score (points) & 77.5 & 0.44 & 0.56 & 0.52 \\
\hline Pin-prick score (points) & 83.5 & 0.50 & 0.67 & 0.45 \\
\hline Comfortable-walking speed $\left(\mathrm{ms}^{-1}\right)$ & 1.0 & 0.86 & 0.89 & 0.90 \\
\hline Maximum-walking speed $\left(\mathrm{ms}^{-1}\right)$ & 1.3 & 0.91 & 0.83 & 0.91 \\
\hline 6-min walking test $(\mathrm{m})$ & 472.5 & 0.91 & 0.89 & 0.94 \\
\hline Walking index for spinal cord injury II & 17.5 & 0.91 & 0.89 & 0.96 \\
\hline
\end{tabular}

Abbreviation: AUC, areas under the curve.

functional recovery because weak, but significant, correlations exist between the LEMS, walking speed and 6MWT $\left(r^{2}=0.24\right.$ and 0.25 , respectively; $P<0.05)$. However, we found that moderate, but significant, correlations existed between the LEMS, walking speed and 6MWT $\left(r^{2}=0.57\right.$ and 0.63 , respectively; $\left.P<0.01\right)$. We suggest that this difference arose because our study included only patients who were capable of walking, whereas the Buehner et al. ${ }^{18}$ study included patients who were unable to walk.

Upper-extremity muscle strength was also an important factor for community ambulation. Waters et al. ${ }^{2}$ reported that upper-extremity strength was often severely compromised and insufficient to enable crutch-assisted walking in patients with tetraplegia, thus accounting for the lower community ambulation rate for patients with incomplete tetraplegia compared with those with paraplegia. The results of this study suggest that the successful community ambulation of patients with cervical ISCI depends on both UEMS and LEMS.

The LEMS and UEMS cutoff points were useful for assessing community ambulation. The AUCs of the LEMS and UEMS suggested a moderate to high accuracy; however, the AUC of the CMAS, LTS and PPS suggested a low accuracy. The LEMS cutoff point in this study was 41.5 , which is higher than the value of $36.9 \pm 7.6$ reported in a previous study. ${ }^{2}$ This may be because in the previous study, 13 of the 23 able to ambulate in the community walked with lower-extremity devices, whereas in this study, only three of the 22 patients used lower-extremity devices. Generally, if the LEMS is high, patients do not require lower-extremity devices. This may be because patients in this study had higher LEMS than the patients in the previous study. The cutoff point of the UEMS in this study was 36.5 , which was higher than that in the previous study $(30.3 \pm 10.8) .^{2}$ This may be because our patients were only graded according to the AIS D, whereas in the previous study, patients were graded according to the AIS C and D. Furthermore, the injury level of our patients (from $\mathrm{C} 4$ to $\mathrm{C} 8$ ) was different from the level of the patients in the previous study (from C3 to C7).

The cutoff points of walking function were useful in assessing community ambulation because the AUC of walking function suggested high accuracy. The comfortable-walking speed cutoff point in this study was $1.00 \mathrm{~m} \mathrm{~s}^{-1}$. This speed was faster than the speed mentioned by van Hedel et al. ${ }^{9}$ (minimum $0.44 \mathrm{~m} \mathrm{~s}^{-1}$ ). We believe that this difference arose from the difference in walking distance between the defined community ambulation in the two studies. The cutoff point of maximum-walking speed in this study was $1.32 \mathrm{~m} \mathrm{~s}^{-1}$. One study found that the speed required to safely cross a street was 
$1.22 \mathrm{~m} \mathrm{~s}^{-1} \cdot{ }^{19}$ Since people can increase their walking speed as required, we consider that it is possible to live in local communities. The WISCI II cutoff point was 17.5 in this study. Similarly, Brotherton et al. ${ }^{10}$ also reported that patients with ISCI who were independent in community ambulation used one cane or a crutch. Therefore, it is clinically useful to know the necessary WISCI II cutoff point for community ambulation.

The median time since injury in this study was 142 days, which was estimated to be within the sub-acute phase after ISCI when recovery is far from completion. However, Spiess et al. ${ }^{20}$ reported that $\sim 90 \%$ of the patients with ISCI who were classified as AIS D within the first 15 days did not convert to AIS E at 12 months post-injury. This was a reason why we were limited to using AIS D patients.

Our findings suggest the importance of assessing upper- and lowerextremity muscle strength, rather than spasticity or sensory, for community ambulation in patients with cervical ISCI who are graded according to the AIS D. The cutoff points of walking function were useful in setting clinical goals for community ambulation.

This study has two limitations. First, only patients graded according to the AIS D were investigated. Our findings may have been different if we had included patients graded according to the AIS C. Second, our sample size was small, and the fact that other parameters, such as balance ${ }^{7}$ or upper-extremity spasticity were not adjusted was a limitation. In order to make general conclusions, further studies examining a larger sample of patients are warranted.

In conclusion, the LEMS and UEMS were the most important factors affecting community ambulation in patients with cervical ISCI who were graded according to the AIS D. The cutoff points of the LEMS, UEMS, comfortable- and maximum-walking speed, 6MWT and WISCI II were $41.5,36.5,1.00 \mathrm{~m} \mathrm{~s}^{-1}, 1.32 \mathrm{~m} \mathrm{~s}^{-1}, 472.5 \mathrm{~m}$ and 17.5, respectively. These cutoff points were of moderate to high accuracy, and we will target these points for walking training in the future.

\section{DATA ARCHIVING}

There were no data to deposit.

\section{CONFLICT OF INTEREST}

The authors declare no conflict of interest.

\section{ACKNOWLEDGEMENTS}

We would like to thank all the volunteers for participating in this study, as well as the staff of the Department of Rehabilitation, Chubu Rosai Hospital.
1 Devivo MJ. Epidemiology of traumatic spinal cord injury: trends and future implications. Spinal Cord 2012; 50: 365-372.

2 Waters RL, Adkins RH, Yakura JS, Sie I. Motor and sensory recovery following incomplete tetraplegia. Arch Phys Med Rehabil 1994; 75: 306-311.

3 Steeves JD, Lammertse D, Curt A, Fawcett JW, Tuszynski MH, Ditunno JF et al. Guidelines for the conduct of clinical trials for spinal cord injury (SCI) as developed by the ICCP panel: clinical trial outcome measures. Spinal Cord 2007; 45: 206-221.

4 Lapointe R, Lajoie Y, Serresse O, Barbeau H. Functional community ambulation requirements in incomplete spinal cord injured subjects. Spinal Cord 2001; 39: 327-335.

5 Robinett CS, Vondran MA. Functional ambulation velocity and distance requirements in rural and urban communities. A clinical report. Phys Ther 1988; 68: 1371-1373.

6 Scivoletto G, Romanelli A, Mariotti A, Marinucci D, Tamburella F, Mammone A et al. Clinical factors that affect walking level and performance in chronic spinal cord lesion patients. Spine 2008; 33: 259-264.

7 Forrest GF, Lorenz DJ, Hutchinson K, Vanhiel LR, Basso DM, Datta S et al. Ambulation and balance outcomes measure different aspects of recovery in individuals with chronic, incomplete spinal cord injury. Arch Phys Med Rehabil 2012; 93: 1553-1564

8 Andrews AW, Chinworth SA, Bourassa M, Garvin M, Benton D, Tanner S. Update on distance and velocity requirements for community ambulation. J Geriatr Phys Ther 2010; 33: 128-134.

9 van Hedel HJEM-SCl Study Group. Gait speed in relation to categories of functional ambulation after spinal cord injury. Neurorehabil Neural Repair 2009; 23: 343-350.

10 Brotherton SS, Saunders LL, Krause JS, Morrisette DC. Association between reliance on devices and people for walking and ability to walk community distances among persons with spinal cord injury. J Spinal Cord Med 2012; 35: 156-161.

11 Catz A, Itzkovich M, Tesio L, Biering-Sorensen F, Weeks C, Laramee MT et al. A multicenter international study on the Spinal Cord Independence Measure, version III: Rasch psychometric validation. Spinal Cord 2007; 45: 275-291.

12 van Middendorp JJ, Hosman AJF, Donders ART, Pouw MH, Ditunno Jr JF, Curt A et al. A clinical prediction rule for ambulation outcomes after traumatic spinal cord injury: a longitudinal cohort study. Lancet 2011; 377: 1004-1010.

13 Ditunno JF, Ditunno PL, Graziani V, Scivoletto G, Bernardi M, Castellano V et al. Walking index for spinal cord injury (WISCI): an international multicenter validity and reliability study. Spinal Cord 2000; 38: 234-243.

14 Kirshblum SC, Burns SP, Biering-Sorensen F, Donovan W, Graves DE, Jha A et al. International standards for neurological classification of spinal cord injury. (revised 2011) J Spinal Cord Med 201134: 535-546.

15 Francis HP, Wade DT, Turner-Stokes L, Kingswell RS, Dott CS, Coxon EA. Does reducing spasticity translate into functional benefit? an exploratory meta-analysis. J Neurol Neurosurg Psychiatry 2004; 75: 1547-1551.

16 Perkins NJ, Schisterman EF. The inconsistency of "optimal" cutpoints obtained using two criteria based on the receiver operating characteristic curve. Am J Epidemiol 2006; 163: 670-675.

17 Waters RL, Adkins R, Yakura J, Vigil D. Prediction of ambulatory performance based on motor scores derived from standards of the American Spinal Injury Association. Arch Phys Med Rehabil 1994; 75: 756-760.

18 Buehner JJ, Forrest GF, Schmidt-Read M, White S, Tansey K, Basso DM. Relationship between ASIA examination and functional outcomes in the NeuroRecovery Network Locomotor Training Program. Arch Phys Med Rehabil 2012; 93: $1530-1540$.

19 Hoxie RE, Rubenstein LZ. Are older pedestrians allowed enough time to cross intersections safely? J Am Geriatr Soc 1994; 42: 241-244.

20 Spiess MR, Mueller RM, Rupp R, Schuld C, van Hedel HJEM-SCI Study Group. Conversion in ASIA impairment scale during the first year after traumatic spinal cord injury. J Neurotrauma 2009; 26: 2027-2036. 